\title{
Estudio sociotécnico de la implementación de un sistema de energía limpia fotovoltaica en las instalaciones de la Fundación Universitaria del Área Andina
}

Carlos Díaz', Leticia Acosta², Lina Buelvas ${ }^{3}$, Keila Barba ${ }^{4}$, Melida Cobo ${ }^{5}$

\section{Resumen}

En la actualidad, la importancia que tiene la generación de fuentes de energía alternativa ha producido un cambio de mentalidad en la sociedad, ya que se promueve la sostenibilidad y responsabilidad ambiental. El objetivo de este proyecto es proporcionar una nueva alternativa que satisfaga las necesidades energéticas en el sector de la cafetería en la Fundación Universitaria del Área Andina mediante el uso de energía fotovoltaica. Esto consiste en una ardua investigación realizada con estadísticas que identifican el servicio que se brindará a la Universidad y, simultáneamente, medir las insuficiencias en el sector ya mencionado. También presenta un estudio detallado del uso de paneles solares como un método de disminución de los costos de energía, que ayudó a obtener los cálculos necesarios para adquirir la resistencia y la duración del tiempo de espera del panel. La reducción de la dependencia energética y la garantía de suministros a través de energías renovables es una necesidad en la sociedad actual, promoviendo la sostenibilidad y responsabilidad ambiental.

Palabras clave: energías renovables, energía solar, sociedad, sostenibilidad ambiental.

\footnotetext{
IIngeniero electrónico, docente del programa Ingeniería Geológica de la Fundación Universitaria del Área Andina, sede Valledupar. Correo: cdiaz5dareandina.edu.co

2Ingeniera electrónica, docente del programa Ingeniería Geológica de la Fundación Universitaria del Área Andina, sede Valledupar. ${ }^{3}$ Ingeniera agroindustrial, docente del programa Ingeniería Geológica de la Fundación Universitaria del Área Andina, sede Valledupar.

4Estudiante del programa Ingeniería Geológica de la Fundación Universitaria del Área Andina, sede Valledupar. ${ }^{5}$ Estudiante del programa Ingeniería Geológica de la Fundación Universitaria del Área Andina, sede Valledupar.
} 


\section{Introducción}

La humanidad con el paso del tiempo ha presentado necesidades que son imprescindibles abastecer y que, debido a diversos aspectos contaminantes, no han podido ser aprovechadas de la mejor manera, incluso teniendo tantos recursos naturales a nuestro favor.

Uno de los recursos más relevantes que puede existir es el de las energías limpias, o también llamadas energías renovables; capaces de generar energía eléctrica a través de fuentes limpias, crecientes e inagotables, a diferencia de aquellos procesos realizados con la ayuda de combustibles fósiles, que son los responsables de grandes consecuencias, como el efecto invernadero, que afecta el clima e incrementa las cifras de emisiones contaminantes en todo el mundo. Dichos mecanismos generadores de energía no solo presentan diferencias en cuanto a la contaminación del entorno, sino también en la diversidad, abundancia y potencial que contiene el aprovechamiento de las energías limpias en cualquier parte del planeta.

La energía solar es una de las clases de producción de energía eléctrica más prometedora, ya que su funcionamiento depende totalmente de los rayos de luz emitidos por el sol. Funciona por medio de paneles compuestos por pequeñas celdas solares encargadas de transformar la luz del sol en electricidad que podemos utilizar para satisfacer actividades cotidianas.

Esta forma de obtención de energía contribuye al desarrollo sostenible y es una fuente muy recursiva y poco costosa a largo plazo.

Es por ello que a nivel internacional, concretamente en las instalaciones del Campus Montecillo del Colegio de Postgraduados, ubicado en el municipio de Texcoco, estado de México, se diseñó y construyó un sistema mecánico-electrónico, para optimizar el proceso de captación de energía solar para su conversión en energía eléctrica, orientando de manera automática paneles solares en dirección de mayor incidencia de radiación, para subsanar la necesidad de luz a sus estudiantes, el mejor rendimiento energético se obtuvo cuando el seguidor solar se orientó al sol cada 60 minutos.

Respecto a la energía generada por un panel fijo, el seguidor solar que se orientó cada 60 min produjo hasta un $27,98 \%$ más de energía, con un gasto energético del $0,3 \%$ de la energía 
total generada o lo que es igual a un $1,3 \%$ de la ganancia energética final. Esto contribuye a la generación de innovación y alcances medio ambientales para la ayuda en cadena de energías limpias en Latinoamérica. (Gómez, Quevedo, Castro, Bravo y Reyes, 2015)

Teniendo en cuenta lo anterior, la Fundación Universitaria del Área Andina actualmente no cuenta con un sistema alternativo de innovación que sea autosostenible y que permita generar energía eléctrica a través de los recursos naturales que se encuentran a nuestros alcances y que, a su vez, permita reducir la contaminación mediante la reducción de emisiones atmosféricas.

Es por ello por lo que la finalidad de esta investigación es implementar o desarrollar este proyecto como beneficio para los estudiantes de la Fundación Universitaria del Área Andina, sede Valledupar, promoviendo el uso de energía fotovoltaica, siendo su principal característica no producir emanación o producción de gases invernaderos, aportando al planeta energía limpia.

En vista de ello, la importancia que recae desde este punto, y teniendo en cuenta que la transformación de la energía genera muchos daños al ambiente, se busca desarrollar un estudio sociotécnico en la implementación de un sistema de energía limpia y fotovoltaica en las instalaciones de la Universidad, de manera que logremos analizar el impacto generado por la implementación de un sistema de paneles solares en la comunidad del barrio Lorenzo Morales de la ciudad de Valledupar, identificar la percepción de la comunidad universitaria sobre la aplicación de sistema de energía limpia, determinar las ventajas de la aplicación de un sistema de paneles solares, diseñar un sistema de energía limpia fotovoltaica e implementar el sistema de energía limpia fotovoltaica en la cafetería de la Fundación Universitaria del Área Andina.

\section{Materiales y métodos}

\section{Localización espacial}

Esta investigación se realizará en torno a la zona de la cafetería de la Fundación Universitaria del Área Andina en el municipio de Valledupar, Cesar, Colombia.

\section{Localización institucional}

Este proyecto se desarrollará bajo 
los criterios y especificaciones de la facultad de Ingeniería y el área de planta física de la Fundación Universitaria del Área Andina Sede Valledupar.

\section{Tipo de investigación}

En la siguiente investigación se plantea un enfoque cuantitativo debido a que este está más centrado en el conteo y clasificación de características y en la construcción de modelos estadísticos y cifras para explicar lo que se observa. Está basado en la toma de datos sobre variables para así poder analizar, en este caso, alternativas de uso de energía, tomando como referencia un previo análisis realizado que relacione los datos concernientes al ahorro total, funcionamiento del sistema y efectividad, y finalmente lograr determinar los beneficios que el mismo traerá a la comunidad.

\section{Método}

Se mostrará de forma clara la finalidad de la investigación mediante el uso de herramientas tales como cuestionarios, encuestas, mediciones y otros equipos para recoger información numérica o medible. Además de presentar marco teórico y textos que sustenten la investigación, se evidencian tablas que contie-

nen información en forma de números y estadísticas, las cuáles hacen más valederos los puntos desarrollados y vuelven la información más verídica.

\section{Materiales}

Los materiales requeridos para la investigación son los siguientes:

- 3 paneles solares de 90 watts

- 2 paneles solares de 150 watts

- 5 baterías de 120 amperios

- 5 reguladores 12/24 10 Amperios digital

- 5 inversores samplex de 450 watts 12 voltios

- 10 tomacorrientes con puerto USB

\section{Procedimiento}

La investigación consta de dos partes, un análisis a través de encuestas para denotar el impacto social de proyectos como estos de energía solar fotovoltaica $y$, a la vez, una evaluación cuantitativa del diseño para todo lo que es referente a la instalación. 


\section{Encuestas}

\section{Establecimiento de los objetivos de la encuesta}

El primer paso para hacer una encuesta consiste en establecer claramente los objetivos que se buscarán alcanzar con esta. Los objetivos de una encuesta se establecen en función a la razón por la que se ha decidido realizarla; por ejemplo, si la razón por la que se ha decidido hacer una encuesta para conocer si la comunidad estudiantil tiene conocimiento de las energías limpias, si se necesita implementar tomas y puertos USB en la universidad. (Crecenegocios.com, 2015)

\section{Determinación de la población 0 universo a estudiar}

Una vez establecido los objetivos, se procede a determinar la población a estudiar. La población o universo por estudiar es el conjunto de personas con características similares de las cuales se desea obtener información que permita, una vez analizada, cumplir con los objetivos de la encuesta. (Couselo et al.)

\section{Identificación de la información a recolectar}

"Una vez determinada la población o universo a estudiar, se procede a identificar la información a recolectar" (Couselo et al.)

\section{Cálculo de la muestra}

El cálculo de la muestra se realiza con la siguiente fórmula:

Fórmula 1. Cálculo de la muestra

$$
u=\frac{N * \partial_{z}+z_{z} * d * b}{z_{z} * d * b * N}
$$

\section{Recolección de la información}

"Una vez diseñado el cuestionario, se procede a recolectar o recoger la información requerida; es decir, a hacer efectiva la encuesta" (Crece Negocios, 2015).

\section{Contabilización y procesamiento de la información}

"Una vez recolectada la información, se procede a contabilizarla y a procesarla de tal manera que pueda ser fácilmente analizada" (Couselo et al.). 


\section{Análisis de la información}

"Finalmente, una vez contabilizada y procesada la información, se procede a analizarla e interpretarla, y a obtener las conclusiones correspondientes". (Couselo et al-).

\section{Diseño del sistema fotovoltaico}

Para el diseño del sistema se utilizan las siguientes fórmulas:

$$
\begin{aligned}
& I=\text { amperio } \\
& V=\text { voltios } \\
& P=\text { watts } \\
& R=\text { ohmnio }
\end{aligned}
$$

Potencia puerto USB

$\mathrm{I}=2 \mathrm{~A}$

$\mathrm{Pot}=\mathrm{VxI}$

Potencia PC portátiles

$$
\mathrm{I}=3 \mathrm{~A}
$$$$
\mathrm{Pot}=\mathrm{VxI}
$$

Potencia total de consumo $=$ Pot $1+$ Pot2 + Pot 3

Calibrado de salida del inversor
Salida de 110 voltios a 450

$$
450 / 110=4,09 \text { amperios }
$$

\section{Paso a paso}

1. Para hacer la base del túnel se recortaron dos láminas galvanizadas de $1,20 \times 55$ y se reforzaron con varillas metálicas de tal forma que se unificaron ambas láminas.

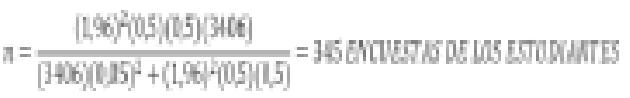

2. Se fijaron las bases de antenas a la estructura. Para este procedimiento se utilizaron chazos metálicos.

3. El panel solar de $90 \mathrm{w}$ se fija a la base, lo cual permite que el panel adquiera una posición perpendicular a la superficie y un ángulo de $45^{\circ}$.

4. Se instalan los píe de amigo de $1 \mathrm{hp}$ en la superficie de la placa.

5. Se ubica la caja de seguridad de $60 \times 40 \times 45$ sobre los píe de amigo. 
6. Se instalan un regulador de 12/24 10 A digital, un inversor Samplex de 450w y 12 voltios, y la batería de $100 \mathrm{~A}$ y 12 voltios, dentro de la caja de seguridad.

7. El panel se conecta al regulador a través de un cable encauchetado número 12 multifilar.

8. Se conecta el regulador a la batería.

9. Se conecta la batería al inversor de $450 \mathrm{w}$ y $12 \mathrm{v}$.

10. Se ubican 9 metros de tubos metálicos galvanizados de $1 / 2$ que inicia desde la placa hasta las zonas verdes, cerca de la zona de bienestar universitario.

11. Se inicia la excavación de una zanja de $40 \mathrm{~cm}$ de profundidad para cumplir la norma.

12. Se ubican seis tubos de PVC de $3 \times 1 / 2$ dentro de la zanja.

13. Se ubica una cajetilla $2 \times 4$ Codelta en la finalización de los tubos para hacer los empalmes.

14. Se ubican 8 tubos metálicos de $3 \times 1 / 2$ con sus respectivas abrazaderas en la parte interna de la cafetería para las cone- xiones de los tomas y lámparas.

15. Se ubican cables unifilares del inversor a la parte inversa y como este solo envía corriente en un solo sentido se tienen que ubicar 36 metros del cable positivo y 36 del cable negativo, por lo tanto, son 72 metros de cable.

16. Se instala la cuchilla para mantener el sistema alimentado por corriente alterna en caso de presentar alguna falla o de falta de corriente por días nublados.

17. Se hace la instalación del cableado interno para la conexión de las lámparas y los tomacorrientes.

18. Se desinstalan las lámparas halógenas antes instaladas para instalar las nuevas lámparas LED de medida $2 \times 32$.

19. Se hacen los empalmes para cada lámpara y para cada toma, cada uno con $2 \mathrm{~m}$ de cable para las conexiones.

20. Se debe hacer el encendido del inversor para hacer la prueba del sistema y se comprueban los voltios que recibe cada toma. 


\section{Resultados}

Las encuestas fueron realizadas tanto en la Urbanización Lorenzo Morales como en la comunidad estudiantil de Areandina. Los cálculos de las muestras para que la encuesta sea válida fueron los siguientes:

\section{Encuestas}

\section{Cálculo de encuesta para la comunidad de Lorenzo Morales}

$z=$ nivel de confianza $(1,96)$

$e=$ grado de error $(0,05)$

$p=$ probabilidad de ocurrencia $(0,5)$
$Q=$ probabilidad de no ocurrencia $(0,5)$

Al aplicar la fórmula 1, tenemos que:

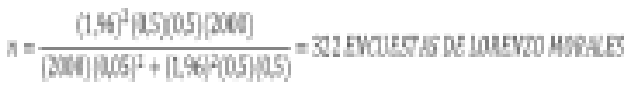

A partir de las encuestas realizadas en la localidad de Lorenzo Morales, se observó que la implementación del sistema de energía fotovoltaica es considerada una contribución significativa a esta comunidad, ya que les permite el uso de hidro bombas y electricidad en sus hogares.

\section{P2}

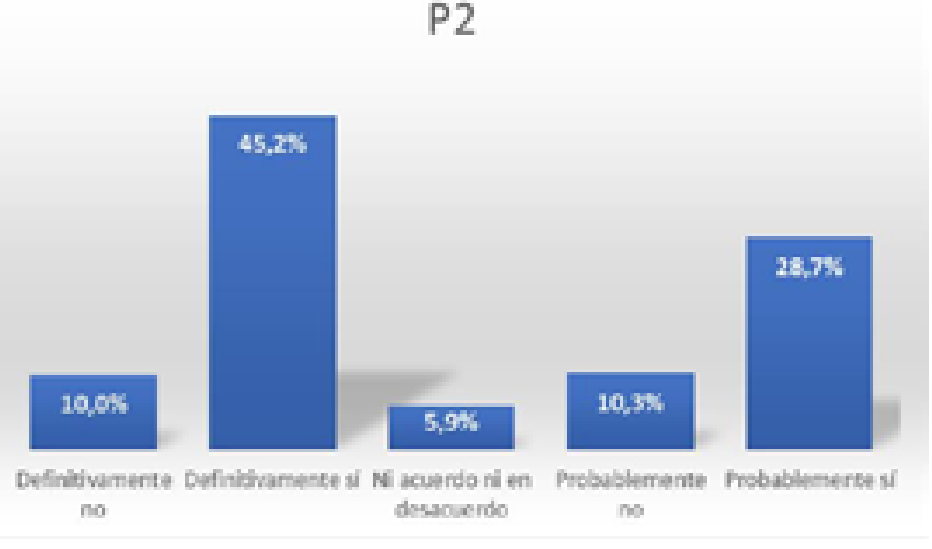

Figura 1. Respuestas de la comunidad acerca del beneficio del sistema. 
Cálculo de encuesta de los estudiantes de la Fundación Universitaria del Área Andina

Este estudio permitió conocer que la Universidad no contaba con un sistema de energías limpias y, de igual forma, los estudiantes desconocían los beneficios de una implementación como esta y su trascendencia.

\section{Cálculos para el diseño del} sistema fotovoltaico
$\mathrm{I}=2 \mathrm{~A}$

Pot $=\mathrm{VxI}$

$\mathrm{V}=5$

$\mathrm{P}=5 \times 2=10 \mathrm{w}$ máximo

Como son dos puertos $10 \mathrm{x} 2=20 \mathrm{w}$

Potencia total de puertos USB $=20 \mathrm{w}$

Potencia PC portátiles

$\mathrm{I}=3 \mathrm{~A}$

Pot $=$ VxI

$\mathrm{V}=19 \mathrm{~V}$

$\mathrm{P}=19 \mathrm{x} 3=57 \mathrm{w}$

$\mathrm{I}=\mathrm{V} / \mathrm{R}$

$\mathrm{V}=\mathrm{P} / \mathrm{I}$

$\mathrm{I}=\mathrm{P} / \mathrm{V}$

Donde

$\mathrm{I}=$ corriente

$\mathrm{V}=$ voltaje

$\mathrm{P}=$ potencia

$\mathrm{R}=$ resistencia

Unidades

$\mathrm{A}=$ amperios

$\mathrm{V}=$ voltios

$\mathrm{P}=$ watts

$\mathrm{R}=$ ohmios

Potencia puerto USB

\section{Potencia Luces LED}

Bombillos leds están entre 10-15 watts

Como son dos tomas de conexión

$57 \mathrm{w} \times 2=114 \mathrm{w}$ total máximo

Potencia total de PC portátiles $=114 \mathrm{w}$

Máximo 15 watts x $2=30$ watts

Potencia máxima de luces led $=30$ watt

Potencial total de consumo $=$ Pot $1+$ Pot2+Pot3

Pot $1=20$ watt

Pot total $=20+114+30=164$ watt

Pot2 $=114$ watt

164 watt $/ 0,75=218.6$ watt para que el 
sistema se sostenga

Pot3 $=30$ watt

Calibre del cable

$90 / 24=3,5$ amperios

$90 / 12=7,5$ amperios

Corriente máxima de regulador que circulará $=7,5$ amperios

Corriente mínima de regulador que circulará $=3,5$ amperios

Calibrado de salida del inversor

Salida de 110 voltios a 450

$450 / 10=4,09$ amperios

\section{Conclusiones}

Esta investigación se realizó con el fin de conocer las energías limpias y lo que representan para la nueva era de las instalaciones y del aprovechamiento de los recursos naturales sin afectar el medio ambiente, siendo uno de los temas más importantes para este nuevo siglo.

Una implementación de este tipo de proyectos representa, no solo para la Universidad, sino también para la ciudad, un gran paso en pro de mejorar las condiciones en las que se encuentra la región, ya que recibe una gran cantidad de radiación, el cual da la seguridad de que dicho proyecto es fiable en esta zona y lleva a sobrepasar las murallas de la institución a nivel regional y nacional, ya que muy pocas instituciones de educación superior en el país han llegado al punto de incursionar en la implementación de métodos de energía alternativa que nos brinden un cuidado del medio ambiente, llevando el proyecto a sectores necesitados, relacionándolo con las comunidades, y siendo pioneros en la fundamentación de proyectos de este tipo.

La culminación de este sistema se llevó acabo en la cafetería de la Universidad. Se puede considerar un gran beneficio para los estudiantes, pues las expectativas generadas por el proyecto fueron suplidas, llevando a los estudiantes a tener un mejor uso de los artefactos tecnológicos en la zona renovable que se encuentra habilitada para todo el personal, la cual se caracterizará por ser un espacio libre y que no generará ingresos al plantel, solo beneficios, convirtiéndolo así en un espacio práctico de estudio y socialización para los estudiantes, centrado en suministrar la importancia que presenta el medio ambiente hoy en día, hecho que es innegable, en especial para la ingeniería como ciencia. El uso y el abuso del medio ambiente y sus energías es cada vez más notorio sobre los complejos fenómenos naturales, altera- 
ciones que están afectando a todos los seres humanos. Es por eso que surge la necesidad de implementar este proyecto, para ser amigables con el planeta, generar una mejor calidad de vida, un mejor ambiente estudiantil con una base ecológica fuerte y expandiendo así la utilización de una energía limpia y renovable dentro del ambiente universitario.

\section{Referencias}

Couselo, R., Williams, E., Pendón, M., Cibeira, N. y Crespi, M. Metodología para la recolección de información primaria para determinar la estructura de financiamiento de pymes del sector industrial y la tasa de rendimiento representativa. Argentina: Universidad Nacional de La Plata.

CreceNegocios (2015). Cómo hacer una encuesta paso a paso. [Página web]. Recuperado de https://www.crecenegocios.com/ comohacer-una-encuesta-paso-apaso/

Creus, A. (2014). "Energía Solar”. En: Ediciones de la U. (Ed.), Energías renovables (pp. 329-377). 2. ${ }^{\circ}$ ed. Bogotá, Colombia: Cano Pina. 